\title{
SEROPREVALENCE OF CYTOMEGALOVIRUS AMONG SOME VOLUNTARY BLOOD DONORS AT THE 37 MILITARY HOSPITAL, ACCRA, GHANA
}

\author{
${ }^{*}$ A.A. ADJEI, H.B. ARMAH AND E.G. NARTER-OLAGA \\ Department of Pathology, University of Ghana Medical School, College of Health Sciences, \\ Korle-Bu, Accra, Ghana.
}

\section{SUMMARY}

Background: Cytomegalovirus (CMV) is one of the most significant pathogens infecting immunosuppressed individuals. CMV is transmissible through transfusion of blood components.

Aim: The goal of this study was to determine the seroprevalence of antibodies to CMV among blood donors seen at the 37 Military Hospital Blood Transfusion Unit, (MHBTU) Accra, Ghana.

Methods: The seroprevalence of antibodies specific for CMV was tested using CMV IgG/IgM particle agglutination test kit and ELISA.

Results: Of the 264 blood donors, 18 were negative and 246 were positive for CMV IgG antibodies, giving an overall CMV prevalence rate of $93.2 \%$. None of the 264 blood donors was positive for CMV IgM antibodies. About $96 \%$ of the donors aged between 30 to 39 years were seropositive for CMV, as against $91.9 \%$ in those aged 20 29 years, $88.6 \%$ in 40 to 49 years, $75.0 \%$ (3 out of 4) in 50 to 59 years, and $100 \%$ (1 out 1) in 60-69 years. There was no statistically significant difference $(\mathrm{P}>0.05)$ in the CMV IgG status in different age groups. The blood donors comprised largely of male donors (236 out of 264), making sex comparisons statistically undesirable. However, all the female $(n=28)$ donors were positive for CMV IgG.

Conclusion: Since about $93 \%$ of blood donors at the MHBTU are seropositive for CMV, it would be very useful to screen blood donors in Ghana for CMV to identify the very few CMV-seronegative blood donors, and maintain an inventory of them for use as donors.

Keywords: Cytomegalovirus, blood donors, seroprevalence.

\section{INTRODUCTION}

Transfusion transmitted disease (TTD) is a major challenge to the transfusion services all over the world. The presence of viruses in blood cells or plasma of asymptomatic donors is the major risk factor for transmitting infectious agents through blood transfusion. Other transfusion transmitted agents include bacteria, mycoplasma, and even protozoa. The main viruses associated with transfusion related infections are hepatitis viruses, retroviruses, and cytomegalovirus (CMV) $)^{1,2}$. The risk of transmitting hepatitis viruses, human retroviruses, such as human immunodeficiency virus (HIV) and human T-lymphotropic viruses types I and II has been reduced drastically by screening for the corresponding antibodies before transfusion.

However, CMV, a member of the human herpes family of viruses, transmissible through blood component transfusions is an important cause of concern world-wide. This is because the majority of adults have serological evidence of previous infections by these viruses. CMV is a ubiquitous agent, and seropositivity rates in the adult population over 40 years of age worldwide are 60 to $100 \%$, possibly due to transmission through breastfeeding, sexual contact and spread from children $^{3,4}$. Like most other herpesviruses, they remain latent in the host after primary infection and persist for lifelong in the organism. Nevertheless, these viruses can be reactivated in immunosuppressed individuals leading to critical outcomes such as interstitial pneumonitis, hepatitis, retinitis, and encephalitis ${ }^{5-7}$. Active infection results in serious morbidity and mortality, and many consider CMV to be one of the most serious pathogens affecting immunosuppressed individuals and an important contribution to intrauterine infection ${ }^{8-10}$. In one study, the relative risk of CMV disease transmission via blood components from seropositive donors was $0.4 \%$ to $12 \%{ }^{11}$. Transfusiontransmitted CMV (TT-CMV) is a significant cause

\footnotetext{
* Author for correspondence
} 
of morbidity and mortality in immunosuppressed patients.

The widespread prevalence of CMV seropositivity makes it difficult for some blood banks to maintain sufficient CMV seronegative blood components for patients at risk for CMV disease. Therefore, the most effective way to minimize the risk of CMV transmission in high-risk recipients would be to administer CMV seronegative blood products or leucoreduced blood products ${ }^{12,13}$. Otherwise, a way out would be to determine the frequency of primary CMV infection among seronegative blood donors ${ }^{14}$. A previous study in Ghana identified CMV infection (cytomegaloviral eosophagitis and enteritis) as one of the major opportunistic infections in Ghanaian HIV-AIDS patients at autopsy ${ }^{15}$, without identifying if the infections in these patients were transfusion-related or not.

The 37 Military Hospital is a 600-bed national referral hospital for military personnel and other soldiers from the West Africa sub-region on peace-keeping missions. According to the Military Hospital Blood Transfusion Unit (MHBTU) estimation, the number of blood units transfused at the Hospital in 2002 was 9,000 units. In view of the large number of transfusions carried out at the Hospital and their great potential for transmitting blood associated pathogens to the recipients, it is extremely necessary that the presence of antibodies to CMV among blood donors be determined. Currently, such a procedure has not been established at the MHBTU. Donor blood is screened only for HIV, hepatitis B virus (HBV), hepatitis C virus (HCV) and syphilis. The present study was undertaken to determine the prevalence of antibodies to CMV infection among blood donors at the MHBTU. Such information may be invaluable to health planners at the hospital and policy makers in Ghana.

\section{SUBJECTS AND METHODS}

This study was carried out between the months of January and April 2004 among blood donors at the MHBTU. This unit serves the military personnel, their dependants, surrounding urban population, and also referred cases from other military personnel from other countries on peace-keeping missions in the West African sub-region. In Ghana, blood donors are volunteers and are also sought from family members of patients and friends needing blood transfusion. They are selected based on the following criteria: age between 18 and 60 years; weight $>45 \mathrm{~kg}$; haemoglobin $>12.5 \mathrm{~g} / \mathrm{dl}$; normal blood pressure [BP], pulse, and body tem- perature; not belonging to any high-risk group (homosexually/heterosexually promiscuous, intravenous drug addicts; patients of sexually transmitted diseases; and no history of any severe current or chronic illnesses). Donors found to be healthy are then permitted to donate blood. Donated blood is routinely screened for HIV 1 \& 2, HBsAg, anti$\mathrm{HCV}$ and syphilis antibodies.

Sera were screened for the presence of antibodies to CMV in parallel with a commercially available CMV IgG/IgM particle agglutination test kit (PAT; Serodia Fujirebio Inc. Japan) and ELISA (anti-HCMV recombinant IgG/IgM; Dade Behring, Marburg, Germany) in accordance with the manufacturer's instructions. Positive and negative standard sera, accompanying the kit were included in each assay. The study was approved by the Ethical and Protocol Review Committee of the University of Ghana Medical School, Accra, Ghana,

Statistical analysis of the data was performed by calculating the Odds Ratio (OR) and the corresponding 95\% confidence intervals in the comparison of the age group having the lowest seropositivity rate with other age groups, and then performing the Chi-square test. $\mathrm{P}$ values $<0.05$ were considered statistically significant.

\section{RESULTS}

Of the 264 blood donors, 236 (89.4\%) were males and $28(10.6 \%)$ were females. Their mean age was 32.1 years (range 19 to 64 years). Sera reactive by both the PAT and ELISA test were considered as positive; and those reactive by either PAT or ELISA test, or neither PAT or ELISA test were considered negative. The combined results showed that 18 were negative (16 were non-reactive by both PAT and ELISA test, and 1 each was reactive to only PAT or only ELISA test) and 246 were positive for CMV IgG antibodies, giving an overall CMV prevalence rate of $93.2 \%$. None of the 264 blood donors were reactive for CMV IgM antibodies by either the PAT or ELISA tests.

Table 1 shows the combined results of CMV IgG seropositivity and age of blood donors. About ninety-six (96\%) percent of the donors (132 out of 138) aged between 30 to 39 years were serpositive for CMV, as against $91.9 \%$ (79 out of 86) in 20 to 29 years, $88.6 \%$ (31 out of 35 ) in 40 to 49 years, and $75.0 \%$ (3 out of 4 ) in 50 to 59 years, and 100\% ( 1 out of 1 ) in 60 to 69 years. There was no statistically significant difference $(\mathrm{P}>0.05)$ in the CMV IgG status in different age groups. The blood do- 
nors comprised largely of male donors (236 out of 264), making sex comparisons statistically undesirable. However, all the female $(n=28)$ donors were positive for CMV IgG.

Table 1 Seroprevalence rate of CMV IgG among blood donors of different age groups in Ghana

\begin{tabular}{ccccc}
\hline Age & $\begin{array}{c}\text { Fre- } \\
\text { quency }\end{array}$ & $\begin{array}{c}\text { Number of } \\
\text { positives } \\
(\%)\end{array}$ & $\begin{array}{c}\text { OR } \\
(\mathbf{9 5 \% C I})\end{array}$ & $\begin{array}{c}\mathbf{P} \\
\text { value }\end{array}$ \\
\hline $20-29$ & 86 & $79(91.9)$ & $1.3(0.6-1.9)$ & 0.13 \\
$30-39$ & 138 & $132(95.7)$ & $1.4(0.8-2.1)$ & 0.09 \\
$40-49$ & 35 & $31(88.6)$ & $1.2(0.5-1.7)$ & 0.15 \\
$50-59$ & 4 & $3(75.0)$ & 1 & - \\
$60-69$ & 1 & $1(100.0)$ & $1.5(0.9-2.4)$ & 0.07 \\
\hline
\end{tabular}

\section{DISCUSSION}

To our knowledge, this is the first report on the seroprevalence of antibodies to CMV among blood donors in the country. The results of this study showed an overall prevalence rate of $93.2 \%$ of CMV IgG among blood donors at the MHBTU, suggestive of ubiquitous past exposure to infection. On the other hand, none of the donors tested positive for CMV IgM, indicating the absence of primary infection. The high seroprevalence rate in adult blood donors reported in this study is comparable to the rates ( $97 \%$ and $96 \%$ ) reported in Tunisia and India, respectively ${ }^{16-18}$. The high prevalence rate in the country indicates the endemicity of infection, and this perhaps could be related to socio-economic, environmental, and climatic factors $^{19}$. In the study reported herein, the seroprevalence of CMV IgG among the blood donors varied with age ranging from $91.9 \%$ in 20 to 29 year, $95.7 \%$ in the 30 to 39 year, to $100 \%$ in the 60 to 69 year age groups (Table 1). The decrease in the percentage seropositivity in the 40 to 49 year and 50 to 59 year age groups is likely due to smaller number of blood donors. Despite this limitation, the results are similar to studies reported elsewhere which showed a significantly increased seropositivity with increasing age of blood donors ${ }^{20,21}$. However, there was no statistically significant difference $(\mathrm{P}>0.05)$ in the CMV IgG serostatus with age, when the other age groups were compared to the 50 to 59 year group with lowest seroprevalence.

The American Association of Blood Banks has recommended transfusion from blood donors who are seronegative for CMV or the use of deglycerolized frozen RBCs whenever transfusion is con- templated in high-risk individuals ${ }^{22}$. These guidelines have helped in drastically minimizing transfusion-transmitted (TT) CMV infection in immunosuppressed recipients in the US. Since about 93\% of blood donors at the MHBTU are seropositive for CMV, it would therefore be very useful to screen blood donors in Ghana for CMV to identify the very few CMV-seronegative blood donors, and maintain an inventory of them for use as donors for immunosuppressed individuals. Furthermore, we propose that the future strategies for the prevention and/or reduction of TT-CMV in Ghana should include the routine screening of donor blood for CMV antibodies as a first step, the subsequent identification of the very few CMVseronegatives, the education and counselling of these CMV-seronegatives on the importance of their status for themselves and the increasing immunosuppressed population and how to maintain their staus, the motivation of these CMV seronegatives for them to become periodic repeat donors, and the maintenance of a database of the epidemiological and contact information of these CMV seronegatives to enable their rapid recall in times of need. This proposed CMV screening of blood donors and the subsequent determination of the actual titres of neutralization antibodies in the numerous CMV-seropositives will ensure the identification of those CMV-seropositives with very high neutralizing antibody titres from whom immunoglobulins can be obtained to treat CMV infections in immunosuppressed individuals, and who will be followed-up and recalled when necessary in the same manner described above for the few CMS-seronegatives.

The above recommendation, though implies a drastic change in blood banking practice in Ghana with financial implications, is timely because transfusion-transmitted CMV (TT-CMV) is a significant cause of morbidity and mortality in immuncsuppressed patients, including premature low-birth weigh infants $(<150 \mathrm{~g})$ born to CMVseronegative mothers and HIV-AIDS patients ${ }^{23}$, whose population increasing in Ghana. Additionally, recent studies have associated chronic CMV infection with prevalent frailty, a state with increased morbidity and mortality in older adults ${ }^{24,25}$. Ghana must introduce the provision of CMV seronegative blood product support to the above stated vulnerable individuals as soon as possible, since this has been the standard of care since the late 1980 s in most developed countries after studies showed this strategy significantly reduced the rate of $\mathrm{TT}-\mathrm{CMV}^{26-28}$. However, the maintenance of CMV-seropositive and CMV-seronegative "dual 
inventories” is expensive, and some countries with high CMV seroprevalence have found it difficult to maintain adequate supplies of CMVseronegative products ${ }^{29}$, as would be the case for Ghana with a 93\% CMV seroprevalence among healthy blood donors herein reported. Thus, alternate methods for the provision of "CMV safe" blood products have been pursued, including the use of leucoreduced blood products which was reported to be comparable to the use of CMVseronegative blood products for the prevention of TT-CMV infection after marrow transplant ${ }^{30}$. However, a more recent study has demonstrated that TT-CMV does occur even after leucoreduction, that CMV-seronegative blood products may thus be superior to leucoreduced blood products, and cautioned against the premature abandonment of the use of CMV-negative inventories particularly for the populations at high risk of and vulnerable to CMV disease for an era of "universal" leucoreduction $^{29}$. It must be noted though that both approaches, of using CMV-seronegative and leucoreduced blood products, may fail and carry a low but definite risk of transmitting CMV infection with "breakthrough" infections occurring at rates as high as 1 to $2 \%^{26-30}$, compared to up to $12 \%$ TT-CMV infection rate when blood products unscreened for CMV antibodies are used ${ }^{11}$. A recent study demonstrated that plasma viremia in seroconverting donors may partially explain the low residual risk of CMV transmission by both CMV-seronegative and leucoreduced CMV- seropositive blood products ${ }^{31}$.

Limitations of the study included the small sample size (which is due to financial constraints in acquiring enough test kits) and the small number of female volunteers presenting at the Blood Bank. Despite this limitation, the general observation is that donated blood at the MHBTU, Accra, Ghana have a high prevalence of antibodies to CMV.

\section{ACKNOWLEDGEMENT}

We would like to thank Staff of the Blood Bank, 37 Military Hospital, Accra, Mr. John Tetteh Ocquaye-Mensah, and Mr. Isaac Boamah, Microbiology Department, U.G.M.S., Korle-Bu, Accra, for their excellent technical assistance and for helpful contributions.

\section{REFERENCES}

1. Herve P. Transfusion safety: emergent or hypothetical risks. Transfus Clin Biol 2000; 7: 30-38.
2. Kuhn J.P. Transfusion-associated infections with cytomegalovirus and other human herpesviruses. Infusionsther Transfusionmed 2000; 27: 138-143.

3. Zhang L., Hanff P., Rutherford C., Churchill C. and Crumpacker C. Detection of human cytomegalovirus DNA, RNA and antibody in normal donor blood. J Infect Dis 1995; 171: 1002-1006.

4. Hecker M., Qiu D., Marquardt K., Bein G. and Hackstein H. Continuous cytomegalovirus seroconversion in a large group of healthy blood donors. Vox Sanguinis 2004; 86: 41-44.

5. Drobyski W., Dume W., Burd E., Knox K., Ash R., Horowitz M., Flomenberg N. and Carrigan D. Human herpesvirus 6 (HHV) infection in allogeneic bone marrow transplant recipients; evidence of a marrow suppressive role for HHV 6 in vivo. J Infect Dis 1993; 167: 735-739.

6. Manez R., Kusne S., Martin M., Linden P., Irish W., Torre-Cisneros J., Kramer D., Ho M. and Starzel T.E. The impact of blood transfusion on the occurrence of pneumonitis in primary cytomegalovirus infection after liver transplant. Transplantation 1993; 30: 594597.

7. Rubin R.H. Impact of cytomegalovirus infections after an organ transplant recipient in bone marrow recipients. Rev Infect Dis 1990; 12: S754-S766.

8. Hillyer C.D., Snydman D.R. and Berkman E.M. The risk of cytomegalovirus infection in solid organ and bone marrow recipients: transfusion of blood components. Transfusion 1990; 30: 659-666.

9. Griffiths P.D., Babboonian C., Rutter D. and Peckham C. Congenital and maternal cytomegalovirus infections in a London population. Br J Obstet Gynaecol 1991; 98: 135140.

10. Wen L., Wu S. and Lu S. The epidemiological study of human cytomegalovirus infection in pregnant women and maternal foetal transmission in three Chinese metropolis. Chung Hua Fuchar Ko Tsa Chih 1996; 31: 714-717. 
11. Krajden M., Shankaran P., Bourke C. and Lau W. Detection of cytomegalovirus in blood donors by PCR using the digene SHARP signal system assay: effects of sample preparation and detection methodology. J Clin Microbiol 1996; 34: 29-33.

12. Roback J.D. CMV and blood transfusions. Rev Med Virol 2002; 12: 211-219.

13. Preiksaitis J.K. The cytomegalovirus-“safe" blood product: is leukoreduction equivalent to antibody screening? Transfus Med Rev 2000; 14: 112-116.

14. Preiksaitis J.K. Prevention of transfusionacquired CMV infection: is there a role for NAT? Transfusion 2003; 43: 302-305.

15. Ayisi N.K., Wiredu E.K., Sata T., Nyadedzor C., Tsiagbe V.K., Newman M., Cofie C.N. and Taneguchi K. T-lymphocytopaenia, opportunistic infections and pathological findings in Ghanaian AIDS patients and their sexual partners. East Afr Med J 1997; 74: 784791.

16. Madhavan H.N., Prakash K. and Agarwal S.C. Cytomegalovirus infections in Pondicherry: a serology survey. Indian J Med Res 1974; 62: 297-300.

17. Kothari A., Ramachandran V.G., Gupta P., Singh B. and Talwar V. Seroprevalence of cytomegalovirus among voluntary blood donors in Delhi, India. J Health Popul Nutr 2002; 20: 348-351.

18. Gargouri J., Elleuch H., Karray H., Rekik H. and Hammami A. Prevalence of anti-CMV antibodies in blood donors in the Sfax region (value in blood transfusion). Tunis Med 2000; 78: 512-517.

19. De Jong M.D, Galasso G.J, Gazzad B., Griffith P.D., Jabs D.A. Kern E.R. and Spector S.A. Summary of the II international symposium on cytomegalovirus. Antiviral Res 1998; 39: 141-162.

20. Galea G. and Urbaniak S.J. Cytomegalovirus studies on blood donors in North-East Scotland and a review of UK data. Vox Sang 1993; 64: 24-30.
21. Gunter K.C. and Luban N.L.C. Transfusion transmitted cytomegalovirus and Epstein-Barr virus diseases. In: Rossi EC, Simon TL, Moss GS, Gould SA editors. Principles of transfusion medicine. Baltimore: Williams and Wilkins, 1996; 717-732.

22. Holland P.V. and Schmitt P.J. Standards for blood banks and transfusion services. $12^{\text {th }}$ ed. Arlington VA: Committee on standards, American Association of Blood Banks. 1987; 30-31.

23. Trincado D. and Rawlinson W.D. Congenital and perinatal infections with cytomegalovirus. J Paediatr Child Hlth 2001; 37: 187-192.

24. Schmaltz H.N., Fried L.P. Xue Q-L., Walston J., Leng S.X. and Semba R.D. Chronic cytomegalovirus infection and inflammation are associated with prevalent frailty in community-dwelling older women. J Am Geriatr Soc 2005; 53: 747-754.

25. High K.P. Chronic infection and frailty: surrogate markers, associations, and causality. $J$ Am Geriatr Soc 2005; 53: 906-908.

26. Bowden R.A., Sayers M., Flournoy N., Newton B., Banaji M., Thomas E.D. and Meyers J.D. Cytomegalovirus immune globulin and seronegative blood products to prevent primary cytomegalovirus infection after marrow transplantation. $\quad N$ Engl J Med 1986; 314: 1006-1010.

27. Miller W.J., McCullough J., Balfour H. H. Jr., Haake R.J., Ramsay N.K., Goldman A., Bowman R. and Kersey J. Prevention of cytomegalovirus infection following bone marrow transplantations: a randomized trial of blood product screening. Bone Marrow Transplant 1991; 7: 227-234.

28. Yeager A.S., Grumet F.C., Hafleigh E.B., Arvin A.M., Bradley J. S. and Prober C.G. Prevention of transfusion-acquired cytomegalovirus infections in newborn infants. J Paediatr 1981; 98: 281-287.

29. Nichols W. G., Price T. H., Gooley T., Corey L. and Boeckh M. Transfusion-transmitted cytomegalovirus infection after receipt of leukoreduced blood products. Blood 2003; 101: 4195-4200. 
30. Bowden R.A., Slichter S.J., Sayers M., Weisdorf D., Cays M., Schock G., Banaji M., Haake R., Welk K., Fisher L. and McCullough J. A comparison of leukocyte-reduced and cytomegalovirus (CMV) seronegative blood products for the prevention of transfusionassociated CMV infection after marrow transplant. Blood 1995; 86: 3598-3603.
31. Drew W.L., Tegtmeier G., Alter H.J., Laycock M.E., Miner R.C. and Busch M.P. Frequency and duration of plasma CMV viremia in seroconverting blood donors and recipients. Transfusion 2003; 43: 309-313. 